\title{
Effect of Volleyball Training Background and Warm-Up Exercise on Passive Kinematics and Musculo-Articular Impedance of the Knee Joint
}

\section{Frédéric Dierick ${ }^{1 *}$, Graziella Laine ${ }^{1}$, Bruno Van Oystaeyen ${ }^{1}$ and Olivier White ${ }^{2,3}$}

${ }^{1}$ Department of Physical Therapy, IESCA Sainte-Thérèse, Haute Ecole Louvain en Hainaut (HELHa), Montignies sur Sambre, Belgium

${ }^{2}$ Université de Bourgogne, Dijon, Campus Universitaire, Unité de Formation et de Recherche en Sciences et Techniques des Activités Physiques et Sportives, France

${ }^{3}$ Institut National de la Santé et de la Recherche Médicale, Unité 887, Motricité-Plasticité, Dijon, France

\begin{abstract}
Knee injuries are common in sport competitions. Risks of injuries can be significantly lowered by warm-up exercise prior to intense activity. This study aimed to determine simultaneously the long-term effect of competitive volleyball training and short-term effect of a warm-up exercise on passive kinematics and musculo-articular impedance of the knee joint. Twelve professional volleyball players (mean age $23.8 \pm 3.9$ years, weight $71.5 \pm 7.1 \mathrm{~kg}$ and height $1.75 \pm$ $0.07 \mathrm{~m}$ ) and 12 moderately active subjects (mean age $20.2 \pm 1.1$ years, weight $63.2 \pm 9.6 \mathrm{~kg}$ and height $1.63 \pm 0.05 \mathrm{~m}$ ) participated in the study. Passive knee joint kinematics (number of oscillations, start and end angles, total duration of oscillations, first four peak flexion angles, first peak extension angle, period of the first three cycles, plateau amplitude, amplitude of first flexion and extension, Flexion and Extension Relaxation Indexes), stiffness and viscosity were investigated using a leg drop pendulum test performed before and after a warm-up exercise consisting of a 10-minute treadmill running. Results showed no effect of competitive volleyball training background on knee joint kinematics, stiffness and viscosity. However, in the moderately active subjects, amplitude of the first flexion and Flexion Relaxation Index were significantly higher after the running exercise than before, reflecting greater musculo-articular compliance of the knee after the warm-up compared to volleyball players. In conclusion, no change in the stiffness of the knee in volleyball players could be beneficial to the practice of volleyball since insufficient lower limb stiffness may destabilize joint motion and increase the incidence of soft tissue injuries.
\end{abstract}

\section{Introduction}

Knee injuries such as anterior cruciate ligament lesions and patellar tendonitis are common in volleyball players. In order to reduce injury risk, many volleyball athletes routinely perform, before maximal exercise, active aerobic exercise warm-up. The main objective of an exercise warm-up is to raise body temperature [1] and this could in turn improve passive biomechanical properties of the musculo-articular systems of the body in regard to external constraints [2].

The global passive mechanical properties of a musculo-articular system depend on anatomical structures crossing the joint. Even if muscles and tendons, the so-called muscle-tendon complex (MTC), contribute significantly to these properties, all other anatomical structures crossing the joint are also involved [3,4]. Preconditioning of the MTC with a warm-up prior to maximal exercise is thought to protect against muscle-tendon injuries [5]. However, to date, the short-term effect of a warm-up exercise on passive kinematics and musculo-articular impedance of the knee joint has never been studied in volleyball players.

The main key objectives of volleyball conditioning are to develop strength and explosive power along with flexibility. Strength is usually developed by resistance training, explosive power by plyometric training and flexibility by static and dynamic stretching. Training and stretching were found to influence the mechanical properties of the MTC. The musculo-tendinous stiffness of Triceps surae is increased by resistance training [6-8] and after plyometric training [9,10]. In other studies, however, no significant modulation of stiffness has been found in the same tendon [6,8]; Grosset et al. [6] even observed a decrease in musculo-tendinous stiffness of Triceps surae after plyometric training. Furthermore, static stretching has been found to reduce passive stiffness of plantar flexor muscles [12], and a significant decrease in stiffness of the Triceps surae tendon was observed after ballistic stretching [13]. On the one hand, the results of the previous studies are equivocal. On the other hand, few studies have focused on the effects of chronic training on the mechanical properties of musculo-articular systems in specific populations of athletes.

Volleyball players are routinely performing plyometrics and stretching in combination with strength training. However, the effects of strength training and stretching on tendon stiffness may be cancelled out, resulting in no reduction in risk of injuries. The purpose of this study was twofold: (1) to determine the long term effect of competitive volleyball training on passive kinematics and musculo-articular impedance of the knee joint and (2) to quantify the short-term effect of a warm-up exercise on the same variables. Passive and musculoarticular impedance of the knee joint results obtained in national-level volleyball players were compared to the results obtained in moderately active subjects, before and after a warm-up exercise consisting of running on a motorized treadmill.

\section{Methods \\ Participants}

Twelve physically active female volleyball players (mean age $23.8 \pm 3.9$ years, weight $71.5 \pm 7.1 \mathrm{~kg}$ and height $1.75 \pm 0.07 \mathrm{~m}$ ) with minimum 7 years experience of playing volleyball participated in the study. Twelve moderately active female participants (mean age 20.2

*Corresponding author: Frédéric Dierick, $\mathrm{PhD}$, Department of Physical Therapy, IESCA Sainte-Thérèse, Haute Ecole, Louvain en Hainaut (HELHa), Rue Trieu Kaisin 134, B-6061 Montignies sur Sambre, Belgium, Tel: +32 712027 90; E-mail: frederic.dierick@gmail.com

Received January 16, 2012; Accepted March 26, 2012; Published March 30 , 2012

Citation: Dierick F, Laine G, Van Oystaeyen B, White O (2012) Effect of Volleyball Training Background and Warm-Up Exercise on Passive Kinematics and MusculoArticular Impedance of the Knee Joint. J Sport Medic Doping Studie S6:001. doi:10.4172/2161-0673.S6-001

Copyright: $\odot 2012$ Dierick F, et al. This is an open-access article distributed under the terms of the Creative Commons Attribution License, which permits unrestricted use, distribution, and reproduction in any medium, provided the original author and source are credited. 
\pm 1.1 years, weight $63.2 \pm 9.6 \mathrm{~kg}$ and height $1.63 \pm 0.05 \mathrm{~m})$ served as controls. The study was conducted in accordance with the Declaration of Helsinki. All subjects were informed of the nature and aim of the study and signed an informed consent form prior to data collection.

Healthy national-level volleyball players were recruited from Charleroi and Farciennes volley clubs (Belgium). They were engaged in strength, plyometric, and stretching training programs, and in highly vigorous physical activity several times per week [14]. They practice physical activity with a mean of $8.7 \pm 2.7$ hours per week. Healthy controls were recruited among students from our physical and occupational therapy departments and had a moderately active lifestyle over the past year, in that they were engaged in light physical activity such as walking or biking for 2-4 hours per week [14] and practiced physical activity with a mean of $2.4 \pm 0.8$ hours per week. Controls were not involved in any strength, plyometric or stretching training programs.

Participants had to meet the following inclusion criteria in order to participate in the study: aged between 18 and 30 years, no current lower limb injury complaints, and no history of neurological disorder. Participants were excluded from the study if they were not able to relax the thigh muscles during the leg drop pendulum test (one control subject and one volleyball player were excluded), had previous history of knee injury or surgery, had patello-femoral instability or had acute quadriceps or hamstrings muscles strain injuries. All participants were explicitly asked to avoid drinking any alcohol or caffeine or do exhaustive exercise during a 24-hour period preceding the experiment.

\section{Muscle EMG recording}

Surface electromyography (sEMG) was used to monitor the activity of Semitendinosus and Vastus Medialis muscles. Before placement of the electrodes, the skin surface was shaved, abraded and cleaned with alcohol in order to reduce skin impedance, and dry-cleaned with ether. An electrically conductive aqueous gel was applied on the electrodes. The electrode placement sites for Semitendinosus and Vastus Medialis muscles were standardized according to the recommendations of Delagi et al. [15]. Electrodes were affixed to the skin with double-sided tape. The ground electrode was placed over the right wrist. Surface EMG activity was recorded at a sampling rate of $1 \mathrm{kHz}$ by bipolar surface sensors (SX 230, Biometrics Ltd, United Kingdom; inter-electrode distance of $20 \mathrm{~mm}$ ). An embedded amplifier in the sEMG sensor (bandwidth $=20-450 \mathrm{~Hz}$; common mode rejection ratio $>96 \mathrm{~dB}$; input impedance $>10 \mathrm{~T} \Omega$; gain=1000) pre-processed the raw signals. Raw sEMG signals were available in real time. Accurate placement of the electrodes was checked before the experiment by asking participants to perform flexion-extension movements of the knee joint against the experimenter's resistance.

\section{Experimental procedure and leg drop pendulum test}

Height, weight, leg length (distance between lateral femoral condyle and lateral malleolus), and mid-thigh circumference (measured on standing subject using a tape placed perpendicular to the long axis of the femur bone at a level halfway between the inguinal fold and the top of the patella) were measured. Body fat composition was determined by bioelectrical impedance analysis using a single-frequency impedance unit (Bodystat 1500 MDD, Bodystat Inc., Douglas, Isle of Man, United Kingdom). Subjects were asked to lie in a supine position for 10 minutes prior to the analysis, with their upper and lower limbs spread slightly. Self-adhesive $\mathrm{Ag} / \mathrm{AgCl}$ surface electrodes (Skintact, RT 34, and Innsbruck, Austria) were attached to the right hand and right foot and crocodile clips were connected to the electrodes. Relevant parameters such as gender, age, height, weight, activity level (very high for volleyball players and low/medium for controls), and waist and hip circumferences were specified to the impedance unit. Only the percentage body fat was collected. Anthropometric and body fat composition characteristics of participants are reported in Table 1.

A leg drop pendulum test quantitatively assessed participants passive knee joint kinematics, stiffness and viscosity before and after a warm-up exercise. During the tests, all blindfolded subjects wore shorts and were barefoot. To minimize any diurnal effects on the results, all measurements were conducted between 8 am and 12 noon. Leg drop pendulum tests were completed by the same investigator in a quiet room with a temperature regulated at $22^{\circ} \mathrm{C}$. The leg drop pendulum test consists in recording free oscillations of a leg-foot segment under the sole action of gravity in relaxed sitting position [16,17]. In the present study, the test was performed with the subject seated at the edge of an examination table with his two leg-foot segments freely hanging at rest, close to the vertical position. The popliteal crease of the knee was placed three finger breadths beyond the edge of the table. The subject's trunk was slightly inclined backwards $\left(10-20^{\circ}\right.$ off the vertical) and leaning onto a cushion to provide a comfortable position that was suitable to allow relaxation of the lower limb muscles. The test was realized on the leg-foot segment of the dominant lower limb (2 left-footed and 10 right-footed in both groups), as determined by the Harris test of lateral dominance [18]. Flexion and extension knee displacements during the test were monitored at a sampling rate of 100 $\mathrm{Hz}$ by an electrogoniometer (SG 150, Biometrics Ldt, Gwent, UK) and a data acquisition system (DataLINK DLK800, Biometrics Ldt, Gwent, UK) connected to a desktop computer equipped with a dedicate data acquisition software (DataLINK software v.3.0, Biometrics Ldt, Gwent, UK). The arms of the electrogoniometer were affixed to the skin of the lateral aspect of the knee with a double-sided tape and the centre of the electrogoniometer was aligned with the centre of the knee joint. Electrogoniometer output was adjusted to zero when the leg-foot segment was hanging at rest, close to the vertical position.

The foot of the subject was placed in a lightweight pulley therapy shoe with Velcro fastening. After lifting the relaxed leg-foot segment from its vertical resting position to the horizontal position (knee fully extended), the investigator attached, by means of a rope, one of the rings of the shoe to a hook screwed in a custom-made wooden suspension system placed in front of the participant. Special attention was drawn as to not lift the thigh from the table when the leg-foot segment was placed in the horizontal position. The rope was then cut, releasing the leg-foot segment that then freely oscillated between flexion and extension until damping stopped the movement. During testing, participants were encouraged to keep electrical activity signals at baseline (relaxed) levels to ensure that measurements were truly passive. Oscillation trials during which electrical activity of muscles increased above baseline (visual inspection) were discarded from the

\begin{tabular}{|l|l|l|l|l|}
\hline & Volleyball & Control & $\mathrm{t} / \mathrm{U}$ & $\mathrm{P}$ \\
\hline Height $(\mathrm{m})$ & $1.75 \pm 0.07$ & $1.63 \pm 0.05$ & -12.69 & $<\mathbf{0 . 0 0 1}$ \\
\hline Weight $(\mathrm{kg})$ & $71.2(65.4-73.7)$ & $60.3(57.7-69.4)$ & 26.00 & $\mathbf{0 . 0 0 9}$ \\
\hline BMI $\left(\mathrm{kg} \mathrm{m}^{-2}\right)$ & $22.5(21.8-24.6)$ & $22.6(21.7-25.2)$ & 69.00 & 0.885 \\
\hline Leg length $(\mathrm{m})$ & $0.45 \pm 0.015$ & $0.41 \pm 0.012$ & -6.05 & $<\mathbf{0 . 0 0 1}$ \\
\hline Mid-thigh circumference $(\mathrm{cm})$ & $53.0(53.0-56.5)$ & $52.5(51.0-55.0)$ & 48.50 & 0.179 \\
\hline Body fat (\%) & $23.3 \pm 5.0$ & $26.5 \pm 4.9$ & 1.59 & 0.125 \\
\hline
\end{tabular}

t: Student t-test, $U$ : Mann-Whitney test; Means \pm SD or Medians (q1-q3) in italic; significant values in bold

Table 1: Anthropometric and body fat composition characteristics of volleyball and control subjects. 
analyses and new trials were acquired. Three successful oscillation trials were recorded per participant and a 30 -second rest interval followed each leg drop pendulum test.

After this test, all subjects realized a warm-up exercise consisting in running on a motorized treadmill (Series 2000; Marquette Electronics; Milwaukee, WI, USA) during 10 minutes at a speed corresponding to $70 \%$ of age-predicted maximal heart rate (208-[0.7xage]) [19]. Immediately after the warm-up, a new leg drop pendulum test was performed exactly as described above.

\section{Signal analysis and biomechanical model}

We measured several kinematic parameters in the successive oscillations observed on the knee joint angle trace over time (Figure

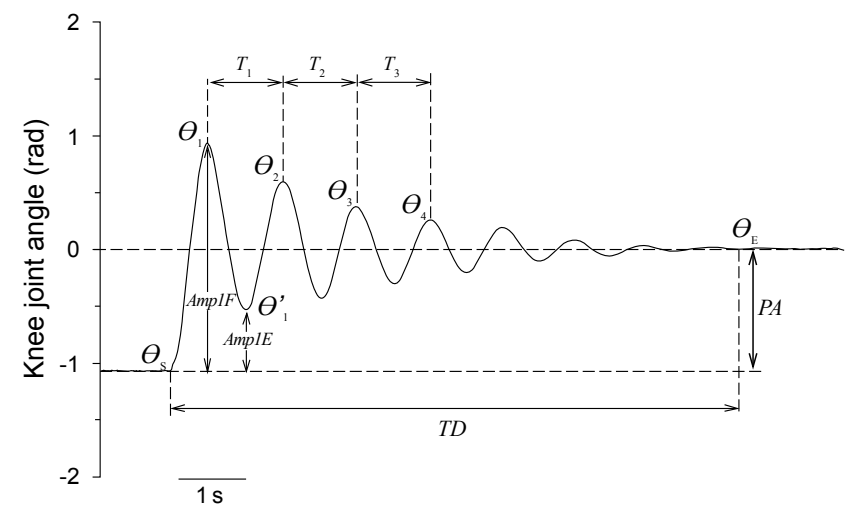

Figure 1: Typical time-knee joint angle plot resulting from a leg drop pendulum test with kinematic parameters. Number of oscillations (NO: 8), angle at the start of the test $\left(\theta_{\mathrm{S}}:-1.06 \mathrm{rad}\right)$, angle at the end of the test $\left(\theta_{\mathrm{E}}: 0.009 \mathrm{rad}\right)$, total duration of oscillations (TD: $8.55 \mathrm{~s})$, first four peak flexion angles $\left(\theta_{1}\right.$ to $\theta_{4}: 0.94$, $0.60,0.38$, and $0.26 \mathrm{rad})$, first peak extension angle $\left(\theta_{1}^{\prime}:-0.53 \mathrm{rad}\right)$, period of the first three cycles ( $T_{1}$ to $T_{3}: 1.10,1.10$, and 1.09s), plateau amplitude (PA: $1.07 \mathrm{rad}$ ), amplitude of first flexion (Amp1F: $2 \mathrm{rad}$ ), and amplitude of first extension (Amp1E: 0.53rad).
1): number of oscillations $(N O)$, start angle $\left(\theta_{\mathrm{S}}\right)$, end angle $\left(\theta_{\mathrm{E}}\right)$, total duration of oscillations (TD), first four peak flexion angles $\left(\theta_{1}\right.$ to $\left.\theta_{4}\right)$, first peak extension angle $\left(\theta_{1}^{\prime}\right)$, period $(T)$ of the first three cycles $\left(T_{1}\right.$ to $\left.T_{3}\right)$, Plateau Amplitude $\left(P A=\theta_{\mathrm{F}}-\theta_{\mathrm{S}}\right)$, amplitude of first flexion $\left(A m p 1 F=\theta_{1}-\right.$ $\left.\theta_{\mathrm{S}}\right)$, amplitude of first extension $\left(A m p 1 E=\theta_{1}{ }_{1}-\theta_{\mathrm{S}}\right)$, Flexion Relaxation Index $(F R I=A m p 1 F / P A)$, Extension Relaxation Index $(E R I=A m p 1 E /$ $P A)$, and ratio $(D)$ between two consecutive peak flexion angles $\left(D_{1}=\theta_{1} /\right.$ $\left.\theta_{2}, D_{2}=\theta_{2} / \theta_{3}, D_{3}=\theta_{3} / \theta_{4}\right)$.

Anthropometric tables of Winter [20] were used to determine leg-foot segment mass $(m)$, distance of the leg-foot segment centre of mass from the knee axis $\left(l_{C M}\right)$, and the radius of gyration of the legfoot segment about the knee axis $(r)$. The sagittal moment of inertia applying to the leg-foot segment rotation around the knee axis $(J)$ was then computed as: $J=m r^{2}$. In addition, some kinematic parameters (see above $D$ with $D_{1}$ to $D_{3}$, and $T$ with $T_{1}$ to $T_{3}$ ) were used, in combination with the anthropometric measurements, to compute viscosity, elasticity, and stiffness of the knee joint. Viscosity $(B)$ and elasticity $\left(K^{\prime}\right)$ were calculated during the first three oscillations $\left(B_{1}\right.$ to $B_{3}$ and $K_{1}^{\prime}$ to $\left.K_{3}^{\prime}\right)$ as $B=\frac{2 J 1 n D}{T}$ and $K^{\prime}=4 \pi^{2} \frac{J}{T^{2}}+\frac{B^{2}}{4 J}$. The stiffness $(k)$ of the knee joint was also computed during the first three oscillations $\left(k_{1}\right.$ to $\left.k_{3}\right)$ as $k=K^{\prime}-m g l_{\mathrm{CM}^{\prime}}$, where $g$ stands for gravitational acceleration $\left(g=9.81 \mathrm{~ms}^{-2}\right)$. Viscosity and stiffness values were normalised by the fifth power of body height $[21,22]$ in order to allow comparison between volleyball players and controls.

\section{Statistical methods}

All results are presented as means \pm SD for the moderately active subjects and volleyball players groups and before and after the warm-up exercise. All statistical procedures were performed with the SigmaStat 3.5 software (SPSS Inc, Chicago, IL, USA) and conducted on relevant parameters.

Assumptions of normality (normal distributions) and homoscedasticity (equal variances) were tested. Student $\mathrm{t}$-tests were realized to compare the mean values between the moderately active

\begin{tabular}{|c|c|c|c|c|c|c|c|c|}
\hline & \multicolumn{2}{|l|}{ Volleyball } & \multirow[b]{2}{*}{$t / W$} & \multirow[b]{2}{*}{$\mathrm{P}$} & \multicolumn{2}{|l|}{ Control } & \multirow[b]{2}{*}{$\mathrm{t} / \mathrm{W}$} & \multirow[b]{2}{*}{$\mathrm{P}$} \\
\hline & Before warm-up & After warm-up & & & Before warm-up & After warm-up & & \\
\hline \multicolumn{9}{|c|}{ Kinematics } \\
\hline NO & $8(7.5-9.5)$ & $9(8-9)$ & 6 & 0.375 & $8 \pm 2$ & $8 \pm 1$ & -0.94 & 0.368 \\
\hline$T D$ & $9.09 \pm 1.5$ & $9.35 \pm 1.4$ & -1.03 & 0.324 & $8.36 \pm 1.8$ & $8.64 \pm 1.6$ & -1.01 & 0.333 \\
\hline$P A$ & $0.89 \pm 0.1$ & $0.90 \pm 0.1$ & -1.23 & 0.245 & $0.90 \pm 0.1$ & $0.92 \pm 0.1$ & -0.89 & 0.394 \\
\hline Amp1F & $1.83 \pm 0.2$ & $1.84 \pm 0.3$ & -0.12 & 0.910 & $1.73 \pm 0.2$ & $1.93 \pm 0.2$ & -6.17 & $<0.001$ \\
\hline Amp1E & $0.41 \pm 0.1$ & $0.36 \pm 0.1$ & 1.28 & 0.226 & $0.45 \pm 0.1$ & $0.45 \pm 0.2$ & -0.07 & 0.948 \\
\hline FRI & $2.07 \pm 0.2$ & $2.07 \pm 0.4$ & -0.02 & 0.984 & $1.94 \pm 0.2$ & $2.12 \pm 0.2$ & -4.16 & 0.002 \\
\hline ERI & $0.46 \pm 0.2$ & $0.41 \pm 0.1$ & 1.23 & 0.244 & $0.47 \pm 0.1$ & $0.48 \pm 0.2$ & -0.16 & 0.873 \\
\hline$D_{1}$ & $1.67 \pm 0.3$ & $1.79 \pm 0.3$ & -1.55 & 0.149 & $1.57(1.5-1.9)$ & $1.64(1.6-1.8)$ & 10 & 0.700 \\
\hline$D_{2}$ & $1.61 \pm 0.4$ & $1.62 \pm 0.3$ & 1.26 & 0.235 & $1.53 \pm 0.2$ & $1.55 \pm 0.2$ & -0.23 & 0.822 \\
\hline$D_{3}$ & $1.56 \pm 0.2$ & $1.62 \pm 0.2$ & -1.48 & 0.168 & $1.63 \pm 0.4$ & $1.58 \pm 0.3$ & 0.98 & 0.349 \\
\hline \multicolumn{9}{|l|}{ Viscosity } \\
\hline$B_{1}$ & $0.42 \pm 0.1$ & $0.48 \pm 0.1$ & -1.96 & 0.076 & $0.29(0.2-0.4)$ & $0.34(0.3-0.4)$ & 13 & 0.577 \\
\hline$B_{2}$ & $0.38(0.3-0.4)$ & $0.34(0.3-0.4)$ & -18 & 0.519 & $0.29 \pm 0.1$ & $0.28 \pm 0.1$ & 0.23 & 0.820 \\
\hline$B_{3}$ & $0.37 \pm 0.1$ & $0.40 \pm 0.1$ & -1.73 & 0.112 & $0.32 \pm 0.2$ & $0.30 \pm 0.1$ & 1.06 & 0.312 \\
\hline \multicolumn{9}{|l|}{ Stiffness } \\
\hline$k_{1}$ & $3.81 \pm 1.1$ & $3.80 \pm 1.2$ & 0.09 & 0.927 & $2.84 \pm 1.0$ & $2.61 \pm 0.8$ & 1.48 & 0.168 \\
\hline$k_{2}$ & $3.64 \pm 0.6$ & $3.66 \pm 0.1$ & -0.12 & 0.911 & $2.55 \pm 0.8$ & $2.61 \pm 0.6$ & -0.47 & 0.648 \\
\hline$k_{3}$ & $3.50 \pm 1.0$ & $3.54 \pm 0.9$ & -0.39 & 0.706 & $2.86 \pm 1.2$ & $2.88 \pm 0.9$ & -0.12 & 0.909 \\
\hline
\end{tabular}

$\mathrm{t}$ : paired t-test, $W$ : Wilcoxon signed-rank test; Means $\pm \mathrm{SD}$ or Medians (q1-q3) in italic; TD expressed in s; $P A$ in rad; $A m p 1 F$ in rad; $A m p 1 E$ in rad; $B_{1}$ to $B_{3}$ in $\mathrm{N} \mathrm{m} \mathrm{s}$ rad ${ }^{-1}$

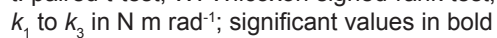

Table 2: Statistical results of warm-up effect on knee joint kinematic variables, stiffness, and viscosity in volleyball players and control subjects. 
subjects and volleyball players groups and non-parametric MannWhitney $U$ tests (before warm-up: ERI, $D_{1}$ to $D_{3}, B_{1}$ to $B_{3}$, and $k_{1}$; after warm-up: $D_{2}, B_{2}, B_{3}$, and $k_{1}$ ) were used when normality test (ShapiroWilk) failed. Paired t-tests were used to compare the mean values before and after the warm-up exercise in each group and non-parametric Wilcoxon signed-rank tests were used when normal distributions were not observed. For all tests, results were regarded as statistically significant at the value of $\mathrm{p}<0.05$.

\section{Results}

Here, we tested whether a running warm-up exercise modifies biomechanical properties of the knee joint in moderately active subjects (controls) and national-level volleyball players. Despite the fact this specific pre-exercise protocol is frequently adopted in competition, its effects on lower limb impedance is unknown. We therefore used the simple yet reliable leg-drop pendulum test to assess passive stiffness of the knee.

Table 2 reports the values of all kinematic, viscosity and stiffness parameters in volleyball players (left columns) and controls (right columns). Within each population category, values are presented before and after warm-up. In the Volleyball player group, all parameters failed to reach significance after warm-up exercise. However, in the moderately active group, two kinematic variables, the amplitude of the first flexion $(A m p 1 F)$ and the Flexion Relaxation Index $(F R I)$ were significantly larger after warm-up exercise (Table $2, \mathrm{t}_{11}=-6.17, \mathrm{p}<0.001$ and $\mathrm{t}_{11}=-4.16, \mathrm{p}=0.002$, respectively). Note that these two variables are directly related by the simple relationship $F R I=A m p 1 F / P A$, where $P A$ was defined as the Plateau Amplitude and didn't show any difference $\left(t_{11} \leq-0.89\right.$ and $p \geq 0.245$ in both groups). Illustration of this effect is depicted in Figure 2. The plotted evolution of knee joint angle over time during a single leg drop pendulum test realised before (dashed line) and after (solid line) the warm-up exercise in a moderately active control participant clearly shows that the amplitude of the first flexion (Amp1F) increased after warming.

\section{Discussion}

The first aim of this study was to determine the long-term effect of competitive volleyball training on passive kinematics and

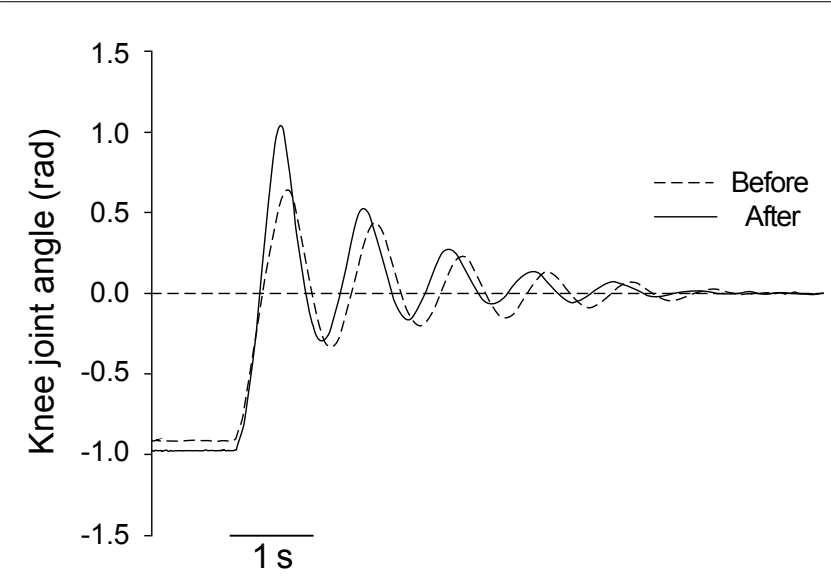

Figure 2: Time-knee joint angle plots resulting from leg drop pendulum tests realised before and after the warm-up exercise in a moderately active control subject (20 years, $60.2 \mathrm{~kg}, 157 \mathrm{~cm}$ ). Before the warm-up exercise, Amp $1 F$ was 1.55 vs 2.02rad after the warm-up. Before the warm-up exercise, $F R I$ was 1.72 vs 2.07 after the warm-up. musculo-articular impedance of the knee joint. In accordance with our hypothesis, the results showed no effect of competitive volleyball training when impedance variables are normalized by taking body height into account. Our hypothesis was that competitive volleyball training will not modify musculo-articular impedance since volleyball players are simultaneously engaged in strength, plyometric, and stretching training programs. Our hypothesis was also supported by a study of Cornu et al. [23] that showed no difference in musculotendinous stiffness of the wrist between chronic trained volleyball players and healthy sedentary subjects. The results of this study show that although protocols specifically studying the effects of resistance training, plyometrics or stretching on the mechanical properties of musculo-articular systems are required, we must also take into account that athletes combine different forms of training and consequently the specific effects may cancel out.

The second aim of this study was to determine the short term-effect of a warm-up exercise on passive kinematics and musculo-articular impedance of the knee joint in competitive volleyball players and moderately active controls. The results showed an effect of warm-up exercise on two kinematic variables of the knee but only among the moderately active controls. The amplitude of the first knee flexion and the Flexion Relaxation Index were significantly larger, reflecting greater musculo-articular compliance of the knee after the warm-up in controls. This phenomenon, although not observed in volleyball players, could be beneficial to the practice of volleyball. Indeed, results from previous studies suggest that insufficient stiffness of the lower limb may destabilize joint motion and increase the incidence of soft tissue injuries [24-26]. Moreover, the maximum power production in jumping would also be improved by an increase in stiffness since a stiffer musculo-articular system might benefit from faster elastic recoil during the upward concentric phase of the jump [27] and a more efficient transfer of force to the skeleton [28]. Also, Grosset et al. [6] observed paired changes in electromechanical delay and musculotendinous stiffness: higher musculo-tendinous stiffness values were associated to lower electromechanical delay values.

During the pendulum test, velocity of downward swing peaked during the first knee flexion after the foot was released from its highest point and when the potential energy of the leg-foot segment was maximal. This could explain why only the amplitude of the first flexion was significantly different after warm-up. The warm-up consisted of a 10-minute treadmill running period. A limitation of our study is that intramuscular temperature of quadriceps muscle was not measured before and after the warm-up, which would have required a somewhat invasive procedure. However, Magnusson et al. [29] observed a significant intramuscular temperature increase within quadriceps after 10 minutes of running on a treadmill at a speed that corresponded to $70 \%$ of maximum $\mathrm{O}_{2}$ uptake and they concluded that 10 minutes of warm-up exercise may be sufficient preparation for muscle performance.

In our study we used a leg drop pendulum test to quantify the effect of volleyball training and warm-up exercise on passive kinematics and musculo-articular impedance of the knee joint. This method allows the estimation of the total stiffness of the knee, including muscles, tendons and all other anatomical structures crossing the joint, such as the skin, subcutaneous tissue, fascia, ligaments, joint capsule and cartilage [4]. Thus, another limitation of our study is that we cannot identify changes in each anatomical structure. However, the advantages of the leg drop pendulum test are that it is non-invasive, easy and quick to use, validated in healthy adults [30] and reliable when rest intervals of 15 seconds are respected between trials [31]. Finally, given the small sample sizes, some 
Citation: Dierick F, Laine G, Oystaeyen BV, White O (2012) Effect of Volleyball Training Background and Warm-Up Exercise on Passive Kinematics and Musculo-Articular Impedance of the Knee Joint. J Sport Medic Doping Studie S6:001. doi:10.4172/2161-0673.S6-001

non-significant results may be related to a lack of statistical power. In our study, the statistical power threshold of 0.8 was not achieved for all variables and negative findings should be interpreted cautiously.

In conclusion, no change in the stiffness of the knee in volleyball players could be beneficial to the practice of volleyball since insufficient lower limb stiffness may destabilize joint motion and increase the incidence of soft tissue injuries. Future work studying the influence of different levels of physical activity should be carried out to test whether simple exercises can improve biomechanical properties of the knee joint in a sedentary group. Inactive people represent an important proportion of the European population; therefore, simple preventive treatments mitigating risks of injury may be a validated recommendation by Public Health organizations.

\section{References}

1. Shellock FG, Prentice WE (1985) Warming-up and stretching for improved physical performance and prevention of sports-related injuries. Sports Med 2: 267-278.

2. McNair PJ, Stanley SN (1996) Effect of passive stretching and jogging on the series elastic muscle stiffness and range of motion of the ankle joint. Br J Sports Med 30: 313-318.

3. Riemann BL, DeMont RG, Ryu K, Lephart SM (2001) The effects of sex, joint angle, and the gastrocnemius muscle on passive ankle joint complex stiffness. J Athl Train 36: 369-375.

4. Wright $V$ (1973) Stiffness: a review of its measurement and physiological importance. Physiotherapy 59: 107-111.

5. Safran MR, Garrett WE Jr, Seaber AV, Glisson RR, Ribbeck BM (1988) The role of warmup in muscular injury prevention. Am J Sports Med 16: 123-129.

6. Grosset JF, Piscione J, Lambertz D, Perot C (2009) Paired changes in electromechanical delay and musculo-tendinous stiffness after endurance or plyometric training. Eur J Appl Physiol 105: 131-139.

7. Kubo K, Kanehisa H, Fukunaga T (2002) Effects of resistance and stretching training programmes on the viscoelastic properties of human tendon structures in vivo. J Physiol 538: 219-226.

8. Kubo K, Morimoto M, Komuro T, Yata H, Tsunoda N, et al. (2007) Effects of plyometric and weight training on muscle-tendon complex and jump performance. Med Sci Sports Exerc 39: 1801-1810.

9. Burgess KE, Connick MJ, Graham-Smith P, Pearson SJ (2007) Plyometric vs. isometric training influences on tendon properties and muscle output. J Strength Cond Res 21: 986-989.

10. Wu YK, Lien YH, Lin KH, Shih TT, Wang TG, et al. (2010) Relationships between three potentiation effects of plyometric training and performance. Scand J Med Sci Sports 20: e80-86.

11. Foure A, Nordez A, Cornu C (2010) In vivo assessment of both active and passive parts of the plantarflexors series elastic component stiffness using the alpha method: a reliability study. Int J Sports Med 31: 51-57.

12. Guissard N, Duchateau J (2004) Effect of static stretch training on neural and mechanical properties of the human plantar-flexor muscles. Muscle Nerve 29: 248-255

13. Mahieu NN, McNair P, De Muynck M, Stevens V, Blanckaert I, et al. (2007) Effect of static and ballistic stretching on the muscle-tendon tissue properties. Med Sci Sports Exerc 39: 494-501.

14. Schnohr P, Scharling H, Jensen JS (2003) Changes in leisure-time physical activity and risk of death: an observational study of 7,000 men and women. Am J Epidemiol 158: 639-644.

15. Delagi EF, lazzetti J, Perrotto A, Morrison D (1994) Anatomical guide for the electromyographer: the limbs and trunk. Charles C. Thomas, Springfield.

16. Wartenberg R (1951) Pendulousness of the legs as a diagnostic test. Neurology
1: $18-24$.

17. Oatis CA (1993) The use of a mechanical model to describe the stiffness and damping characteristics of the knee joint in healthy adults. Phys Ther 73: 740749.

18. Harris AJ (1953) Harris Tests of Lateral Dominance. New York, NY: The Psychological Corporation.

19. Tanaka H, Monahan KD, Seals DR (2001) Age-predicted maximal heart rate revisited. J Am Coll Cardiol 37: 153-156.

20. Winter DA (1979) Biomechanics of Human Movement. John Wiley and Sons, Chichester.

21. Lebiedowska MK, Syczewska M, Graff K, Kalinowska M (1996) Application of biomechanical growth models of the quantitative evaluation of the motor system in children. Disabil Rehabil 18: 137-142.

22. Valle MS, Casabona A, Sgarlata R, Garozzo R, Vinci M, et al. (2006) The pendulum test as a tool to evaluate passive knee stiffness and viscosity of patients with rheumatoid arthritis. BMC Musculoskelet Disord 7: 89.

23. Cornu C, Maïsetti O, Ledoux I (2003) Muscle elastic properties during wrist flexion and extension in healthy sedentary subjects and volley-ball players. Int J Sports Med 24: 277-284.

24. Kuitunen S, Kyrolainen H, Avela J, Komi PV (2007) Leg stiffness modulation during exhaustive stretchshortening cycle exercise. Scand J Med Sci Sports 17: $67-75$.

25. Flanagan EP, Harrison AJ (2007) Muscle dynamics differences between legs in healthy adults. J Strength Cond Res 21: 67-72.

26. Butler RJ, Crowell HP 3rd, Davis IM (2003) Lower extremity stiffness: implications for performance and injury. Clin Biomech (Bristol, Avon) 18: 511517.

27. Arampatzis A, Schade F, Walsh M, Bruggemann GP (2001) Influence of leg stiffness and its effect on myodynamic jumping performance. J Electromyogr Kinesiol 11: 355-364.

28. Wilson AM, Watson JC, Lichtwark GA (2003) Biomechanics: A catapult action for rapid limb protraction. Nature 421: 35-36.

29. Magnusson SP, Aagaard P, Larsson B, Kjaer M (2000) Passive energy absorption by human muscle-tendon unit is unaffected by increase in intramuscular temperature. J Appl Physiol 88: 1215-1220.

30. Brown RA, Lawson DA, Leslie GC, Part NJ (1988) Observations on the applicability of the Wartenberg pendulum test to healthy, elderly subjects. J Neurol Neurosurg Psychiatry 51: 1171-1177.

31. Bohannon RW (1987) Variability and reliability of the pendulum test for spasticity using a Cybex II isokinetic dynamometer. Phys Ther 67: 659-661. 